\title{
Design of Centralized Fractional order PI Controller for Two Interacting Conical Frustum Tank Level Process
}

\author{
S. K. Lakshmanaprabu ${ }^{1 \dagger}$, A. Wahid Nasir ${ }^{2}$ and U. Sabura Banu ${ }^{1}$ \\ ${ }^{1}$ B S Abdur Rahman Crescent University, Chennai, Tamilnadu, 6000048, India \\ ${ }^{2}$ National Institute of technology Jamshedpur, Jharkhand, 831014, India.
}

†Corresponding Author Email: prabusk.leo@gmail.com

(Received February16, 2017; accepted July 30, 2017)

\begin{abstract}
The mathematical model for two interacting conical frustum tank level (TICFTL) process is proposed. The control of TICFTL process is difficult due to the nonlinearity, interaction effect between input flow and output level of tanks. Hence, the nonlinear process is linearised around proper operating points. Then, the decentralized PI, centralized PI, centralized FOPI controllers are designed and then the control parameters are tuned using genetic algorithm. The closed loop controller performances are simulated and compared in terms of settling time, rise time and integral error criteria. It is found that centralized PI controller has better servo and regulatory response than the decentralized PI, centralized FOPI controller. The liquid inflow rates are optimally manipulated by the centralized PI controller to track the set point variation and to reject the disturbance effectively than centralized FOPI, decentralized PI controller.
\end{abstract}

Keywords: Two interacting conical frustum tank level process; Flow control; Level control; Centralized PI; FOPI; Decentralized PI controller.

\section{NOMENCLATURE}

$\begin{array}{ll}\mathrm{a}_{1}, \mathrm{a}_{2}, \mathrm{a}_{12} & \begin{array}{l}\text { cross sectional area of pipe } \\ \mathrm{dt}\end{array} \\ g & \text { time step } \\ G p(s) & \text { gravity } \\ h_{1}, h_{2} & \text { liquid level in tank } 1,2 \\ \mathrm{H}_{1}, \mathrm{H}_{2} & \text { maximum height of tank } 1,2 \\ I V_{1}, V_{2} & \text { input voltage to pump } 1,2 \\ K p p_{1} & \text { pump gain of pump 1 }\end{array}$

\section{INTRODUCTION}

Controlling of liquid level and flow in process tanks are the challenging control problem in the process industries. Generally, the liquids are pumped and stored in the tanks for processing; again it is pumped to other tanks for other operations. The conical tanks are widely used in liquid treatment industry, concrete industry and hydrometallurgical industries (Ravi et al. 2014). The TICFTL process is a typical two input two output (TITO) process which exhibits nonlinear characteristics and dynamic coupling effect between inputs and outputs. The control of TITO process requires dedicated multiloop or multivariable control

\author{
$\mathrm{Kpp}_{2} \quad$ pump gain of pump 2 \\ $\mathrm{R}_{1}, \mathrm{R}_{2}$ top Radius of conical frustum tank \\ $\mathrm{r}_{\text {in } 1}, \mathrm{r}_{\text {in2 }}$ bottom radius of tank 1,2 \\ $\beta_{1} \quad$ valve co-efficient of tank1 outlet \\ $\beta_{12} \quad$ valve co-efficient of interaction pipe \\ $\beta_{2} \quad$ valve co-efficient of tank2 outlet \\ $\gamma G c(s) \quad$ controller transfer function \\ $\lambda$ order of FOPI controller
}

system. Commonly, the process industries employ multiloop PID controller because of its simple structure, robustness and failure tolerance (Astrom et al. 2002). The multiloop PID controllers produce better control performance for the system with modest interaction. But it fails to provide desirable control performance for the system with severe interaction effect between inputs and outputs. Such a highly coupled multivariable system requires a decoupler based centralized control or multivariable centralized controller scheme for compensating the interaction disturbances effectively. These two types of centralized control (full matrix control) strategies are employed with PI/PID controller for enhancing the servo tracking and regulatory 
response of controller performance.

Vijay kumar et al. (2012) developed the centralized PI controller for TITO process. Yuling Shen et al. (2014) Xiaoli Luan et al. (2014) have proposed full matrix controller based on the effective open loop transfer function model. Juan Garrido et al. (2012) have developed multivariable centralized control design using inverted decoupling method. Wieder chang et al. (2007) has developed multivariable PID controller for TITO process using genetic algorithm with modified cross over formula. In this approach, the IAE is considered as the objective function and then the centralized PID controller 12 tunable parameters are tuned using GA. The effectiveness of modified GA based PID controller is compared with BLT method based PID controller and traditional GA based PID controller. Ravi V R et al. (2014) have developed multiloop PI controller with decoupler for two interacting conical tank level process. The multiloop PI controller tuned using stability boundary equation method and genetic algorithm.

The past two decades fractional calculus has been gained popularity in science and engineering application, especially in control theory. Podlubny et al. (1999) introduced generalization of PID controller called FOPID controller with additional degree of freedom to enhance the robustness of closed loop system. In the process control industry, more than $90 \%$ of the controller loops are controlled by PI/PID controller. However, many authors have demonstrated the advancement of FOPID controller in closed loop control system. The additional non-integer order integration and differentiation operator makes FOPID more robust than integer order PID control. The additional two parameters in FOPID controller introduced complexity in the controller tuning. Various analytical based methods are reported in the literature. However, the optimization based tuning methods have gained popularity in fractional order control design.

The global optimization such as genetic algorithm is widely used to solve complex engineering problems. The GA has been utilized in many control engineering problem to obtain optimal tuning parameters to provide desirable control performance.

Puneet mishra et al. (2015) designed fractional order fuzzy PID control for binary distillation process (TITO process) where the FOPID controller parameter tuned using GA with weighted sum of integral sum of absolute error as an objective function. Morteza moradi (2014) proposed multivariable FOPID control for TITO process where the 20 FOPID controller tuning parameters are tuned using modified GA with weighted sum of IAE as an objective function. It was claimed that multivariable FOPID controller provides better performance than $\mathrm{H}_{\infty}$ synthesis based FOPID controller. Conception A monje et al. (2004) tuned FOPI controller using iteration based optimization with robust specifications. The phase margin, gain margin and robustness to plant gain variation are considered as the constraints of the optimization and then FOPI controller are tuned to meet these three specifications. pan I and das S (2013) has designed multiloop FOPID controller for bench mark TITO process using particle swarm optimization algorithm. In which, the multiloop FOPID controller parameters obtained by optimizing the time multiplied squared error of both loops. Priya c et al. (2014) has designed multiloop PI control and fractional order PI control for spherical-conical interconnected tank systems.

The decoupled control scheme and multivariable centralized controller are designed with linear PI/PID controller, which are failed to produce reasonable performance for the nonlinear system. Hence, the adaptive PI/PID control has been designed for nonlinear MIMO systems by combining family of linear PI/PID controller with gain scheduling scheme. Ananda natrajan et al. (2006) has designed PI controller for single conical tank process, where the multimodel based gain scheduler and neural network based gain scheduler are utilized for controlling the entire operating regimes of conical tank process. Many adaptive mechanisms have been presented for nonlinear process, in which family of linear controller with gain scheduler is extensively used as adaptive mechanism. The conventional gain schedule may change the PID controller parameter abrupt across the boundaries of the operating regions. But the fuzzy logic based gain scheduling method utilizing bumpless transfer function to adjust the controller parameter smoothly (Balametee TP et al. 2000).

Zhao Z-Y et al. (1993) have demonstrated the fuzzy gain scheduler based PID control for process control application. It has been claimed that fuzzy logic based gain scheduler provides satisfactory control performance for nonlinear system (Dhanalakshmi Vinodha $\mathrm{R}$ et al. (2013)). An adaptive fuzzy based gain scheduler method is developed to provide pre-specified control objectives for nonlinear system. Vijayalakshmi et al. (2014) used multimodal based gain scheduler to control the level of liquid in the single conical tank process, where the family of linear PID controller parameters is found for three operating regimes and then weighted scheduler is designed to adjust the controller parameter based on the operating regimes. Nithya et al. (2008) has developed fuzzy logic controller for nonlinear spherical tank level process. In that, fuzzy logic rule base is tuned using genetic algorithm. It has been claimed that fuzzy logic control is superior than the conventional PI control. Blanchett et al. (2000) has designed improved fuzzy gain scheduler to enhance the PID control performance for nonlinear process. The comparative results of fuzzy logic gain scheduler PID with model predictive control is demonstrated. Kamala et al. (2012) has demonstrated fuzzy gain scheduler based PID controller for nonlinear MIMO process. Almeida otacilio da et al. (2002) has developed fuzzy rules for MIMO system based on the information of human expertise about the gain 


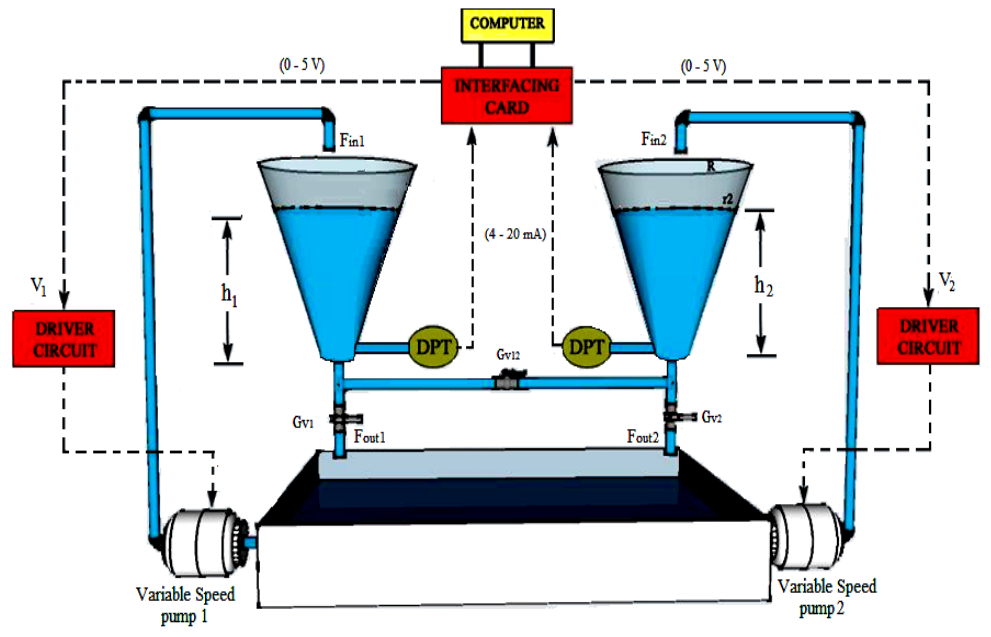

Fig. 1. Schematic diagram of Two Interacting Conical Frustum tank level (TICFTL) process.

and phase margin of the specific multivariable closed loop system.

The objective of this work is to develop a mathematical model for proposed TICFTL process and then to design an optimal closed loop control system to control the liquid level of tank 1, tank 2 . In this paper, genetic algorithm is used to found the Decentralized PI, centralized PI, FOPI controller parameters for minimum value of integral error indices. The controller parameters are tuned to overcome the interaction effect between loops and to improve the servo, regulatory performance of closed loop system.

This paper organized as follows. The mathematical model for proposed TICFTL process is briefly explained in section 2. The decentralized PI controller design procedure is given in section 3 . The centralized PI, FOPI control scheme and genetic algorithm based tuning procedures are detailed in section 4 . The simulation results of controller are analyzed in section 5. Finally the conclusion of proposed work is highlighted in section 6 .

\section{TWO INTERACTING FRUSTUM CONICAL TANK PROCESS DESCRIPTION}

The proposed system consists of two interacting conical frustum tanks connected by interacting pipe. The heights of tanks are $50 \mathrm{~cm}$ and top and bottom radius of conical tanks are $40 \mathrm{~cm}$ and $14 \mathrm{~cm}$. The gate values $\mathrm{Gv}_{1}, \mathrm{Gv}_{2}$ and interaction valve Gv12 are partially opened and kept fixed. The interaction effect of process can be changed by the hand value Gv12. The two tanks getting inflow of liquid from variable speed pumps. The manipulated inputs of system are the voltage applied to the pumps. The range of input voltage is 0 to $5 \mathrm{~V}$, which is directly proportional to rate of change of inflow. The differential pressure transmitter used for measuring the level in terms of milliamps. The main aim is to control the liquid level in the tanks by manipulating the applied input voltages to motor pumps 1 and 2.

\subsection{Mathematical Modeling of TICFTL Process}

The mathematical model of TICFTL process is derived from the mass balance equation. The single conical frustum tank system shown in Fig. 2.

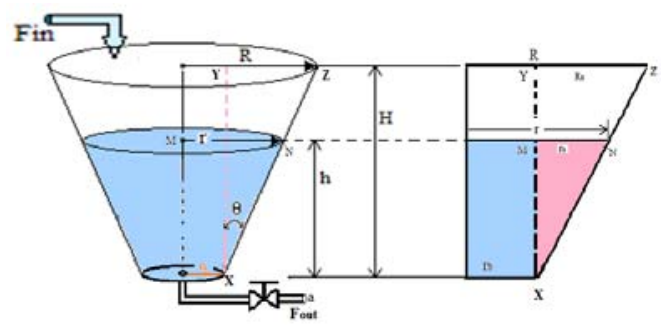

Fig. 2. Volume of liquid in the frustum tank.

The mathematical model for single frustum conical tank process is derived using the conservation of mass and Bernoulli's principle as follows,

Rate of accumulation $=$ Rate of inflow - Rate of Outflow

$\frac{d V o l}{d t}=$ Fin - Fout

where Vol is a volume of liquid in the cone frustum tank. The volume of liquid change due its varying surface area of the tank. The Volume of cone frustum tank $\mathrm{Vol}$ is

$V o l=\frac{\pi}{3}\left(r_{i n}^{2}+r^{2}+r_{i n} r\right)$

where $r_{\text {in }}$ bottom radius of tank and $r$ is the top radius of liquid. The varying top radius of liquid level is found using trigonometric law.

$\tan \theta=\frac{N M}{X N}=\frac{Y Z}{X Y}$

where $\theta$ is the angle of frustum conical slope. 
S. K. Lakshmanaprabu et al. / JAFM, Vol. 10, Special Issue, pp. 23-32, 2017.

Table 1 Operating point conditions

\begin{tabular}{|c|c|c|c|c|}
\hline \multirow[t]{2}{*}{ Region } & \multicolumn{2}{|c|}{$\begin{array}{l}\text { Operating point } \\
\text { Fin } 1 \mathrm{Vs} \text { h1 }\end{array}$} & \multicolumn{2}{|c|}{$\begin{array}{l}\text { Operating point } \\
\text { Fin2 Vs h2 }\end{array}$} \\
\hline & Fin1 & h1 & Fin 2 & $\mathrm{~h} 2$ \\
\hline 1 & $(0-1.5) \mathrm{V}$ & $0-4.67$ & $(0-1.5) \mathrm{V}$ & $0-4.67$ \\
\hline 2 & $(1.5-3) \mathrm{V}$ & $4.42-11.62$ & $(1.5-3) \mathrm{V}$ & $4.42-11.62$ \\
\hline 3 & $(3-5) \mathrm{V}$ & $11.62-27.26$ & $(3-5) \mathrm{V}$ & $11.62-27.26$ \\
\hline
\end{tabular}

Table 2 The transfer function matrix for regime (Tank 1/Regime 1, 2, 3 ; Tank 2/Regime 1, 2)

\begin{tabular}{|c|c|c|c|c|}
\hline \multirow[b]{2}{*}{$\begin{array}{c}\text { Tank } \\
1\end{array}$} & \multicolumn{4}{|c|}{ Tank 2} \\
\hline & \multicolumn{2}{|c|}{ Regime 1} & \multicolumn{2}{|c|}{ Regime 2} \\
\hline \multirow{4}{*}{1} & $0.157 s+0.03073$ & 0.02085 & $0.1417 s+0.005834$ & 0.002961 \\
\hline & $s^{2}+0.393 s+0.02109$ & $\overline{s^{2}+0.393 s+0.02109}$ & $s^{2}+0.0904 s+0.001561$ & $\overline{s^{2}+0.0904 s+0.001561}$ \\
\hline & 0.02085 & $0.1573 s+0.0309$ & 0.002961 & $0.1323 s+0.006514$ \\
\hline & $\overline{s^{2}+0.393 s+0.02109}$ & $\overline{s^{2}+0.393 s+0.02109}$ & $\overline{s^{2}+0.0904 s+0.001561}$ & $\overline{s^{2}+0.0904 s+0.001561}$ \\
\hline \multirow{4}{*}{2} & $0.1323 s+0.00651$ & 0.00296 & $0.117 s+0.0131$ & 0.01146 \\
\hline & $\overline{s^{2}+0.0904 s+0.00156}$ & $\overline{s^{2}+0.0904 s+0.00156}$ & $\overline{s^{2}+0.2237 s+0.002914}$ & $\overline{s^{2}+0.2237 s+0.002914}$ \\
\hline & 0.002961 & $0.1417 s+0.005834$ & 0.01146 & $0.117 s+0.0131$ \\
\hline & $\overline{s^{2}+0.0904 s+0.00156}$ & $\overline{s^{2}+0.0904 s+0.00156}$ & $\overline{s^{2}+0.2237 s+0.002914}$ & $\overline{s^{2}+0.2237 s+0.002914}$ \\
\hline \multirow{4}{*}{3} & $0.095 s+0.00242$ & 0.0009032 & $0.0811 s+0.0015$ & 0.00089 \\
\hline & $\overline{s^{2}+0.0414 s+0.000338}$ & $\overline{s^{2}+0.0414 s+0.000338}$ & $\overline{s^{2}+0.03497 s+0.000193}$ & $\overline{s^{2}+0.03497 s+0.000193}$ \\
\hline & 0.0009032 & $0.1227 s+0.001966$ & 0.00089 & $0.0886 s+0.00145$ \\
\hline & $\overline{s^{2}+0.0414 s+0.000338}$ & $\overline{s^{2}+0.0414 s+0.000338}$ & $\overline{s^{2}+0.03497 s+0.000193}$ & $\overline{s^{2}+0.03497 s+0.000193}$ \\
\hline
\end{tabular}

$\mathrm{NM}=\mathrm{r}_{\mathrm{s}}$, is an incremental radius of liquid level due to slope surface. $r_{S}=\frac{R_{S}}{H} h=\frac{\left(R-r_{i n}\right)}{H} h$.

The top radius of liquid level, $r=r_{\text {in }}+r_{s}$;

$r=r_{i n}+\frac{\left(R-r_{i n}\right)}{H} h$.

After substituting ' $r$ ' value in Eq. 2, the volume of liquid in cone frustum becomes,

$V o l=\frac{\pi}{3}\left[3 r_{i n}^{2} h+3 r_{i n}\left(\frac{R-r_{i n}}{H}\right) h^{2}+\left(\frac{R-r_{i n}}{H}\right)^{2} h^{3}\right]$

After substituting Eq. 5 in Eq. 1,

$$
\frac{d h}{d t}=\frac{F_{i n}-\beta a \sqrt{2 g h}}{\frac{\pi}{3}\left[3 r_{i n}^{2}+6 r_{i n}\left(\frac{R-r_{i n}}{H}\right) h+3\left(\frac{R-r_{i n}}{H}\right)^{2} h^{2}\right]}
$$

Where $F_{\text {out }}=\beta a \sqrt{2 g h}$, ' $\mathrm{a}$ ' is a cross sectional area of outlet pipe and $\beta$ is the ratio of gate valve opening ( $\beta$ varies from 0 to 1 ). When the valve is fully closed, $\beta$ is 0 , when the valve is fully open $\beta$ is 1 . V is input voltage, Kpp is the pump gain.

Similarly, the mathematical model for TICFTL process is developed,

$$
\frac{d h_{1}}{d t}=\frac{\mathrm{k}_{\mathrm{pp} 1} \mathrm{~V}_{1}-\beta_{1} a_{1} \sqrt{2 g h_{1}}-\operatorname{sign}\left(h_{1}-h_{2}\right) \beta_{12} a_{12} \sqrt{2 g\left|h_{1}-h_{2}\right|}}{\frac{\pi}{3}\left[3 r_{i n 1}^{2}+6 r_{i n 1}\left(\frac{R_{1}-r_{i n 1}}{H_{1}}\right) h_{1}+3\left(\frac{R_{1}-r_{i n 1}}{H_{1}}\right)^{2} h_{1}^{2}\right]}
$$

$$
\frac{d h_{2}}{d t}=\frac{\mathrm{k}_{\mathrm{pp} 2} \mathrm{~V}_{2}+\operatorname{sign}\left(h_{1}-h_{2}\right) \beta_{12} a_{12} \sqrt{2 g\left|h_{1}-h_{2}\right|}-\beta_{2} a_{2} \sqrt{2 g h_{2}}}{\frac{\pi}{3}\left[3 r_{i n 2}^{2}+6 r_{i n 2}\left(\frac{R_{2}-r_{i n 2}}{H_{2}}\right) h_{2}+3\left(\frac{R_{2}-r_{i n 2}}{H_{2}}\right)^{2} h_{2}^{2}\right]}
$$

The flow rates are function of applied input voltage.

The tanks are identical tanks, so the bottom radius $r_{\text {in } 1}=r_{\text {in } 2}=14 \mathrm{~cm}$, top radius $R_{1}=R_{2}=20 \mathrm{~cm}$. The $H_{1}, H_{2}$ are the height of frustum conical tank $\left(\mathrm{H}_{1}, \mathrm{H}_{2}=50\right.$ $\mathrm{cm})$. The $\mathrm{h}_{1}, \mathrm{~h}_{2}$ are the liquid level of tank 1,2 . The valve coefficient also same for both tanks (i.e, $\beta_{1}=$ $\left.\beta_{2}=0.33\right)$ and interaction pipe valve coefficient $\beta_{12}=0.2$.

The TICFTL process exhibits nonlinear characteristic, hence the operating regimes are found using piecewise linearization method for controller design. The operating points obtained from input-output characteristic and tabulated in the Table.3. The $\mathrm{k}_{\mathrm{pp} 1}, \mathrm{k}_{\mathrm{pp} 2}$ are the pump 1, 2 gains (25 
S. K. Lakshmanaprabu et al. / JAFM, Vol. 10, Special Issue, pp. 23-32, 2017.

Table 3 The transfer function matrix for (Tank 1/Regime 1, 2, 3; Tank 2 / Regime 3)

\begin{tabular}{|c|c|}
\hline & Tank 2 \\
\hline Tank1 & Regime 3 \\
\hline Regime 1 & {$\left[\begin{array}{ll}\frac{0.1227 s+0.001966}{s^{2}+0.0414 s+0.0003378} & \frac{0.0009032}{s^{2}+0.0414 s+0.0003378} \\
\frac{0.0009032}{s^{2}+0.0414 s+0.0003378} & \frac{0.095 s+0.00242}{s^{2}+0.0414 s+0.0003378}\end{array}\right]$} \\
\hline Regime 2 & {$\left[\begin{array}{ll}\frac{0.0886 s+0.001457}{s^{2}+0.03497 s+0.0001935} & \frac{0.000894}{s^{2}+0.03497 s+0.0001935} \\
\frac{0.000894}{s^{2}+0.03497 s+0.0001935} & \frac{0.08116 s+0.001503}{s^{2}+0.03497 s+0.0001935}\end{array}\right]$} \\
\hline Regime 3 & {$\left[\begin{array}{ll}\frac{0.0121 s+0.002686}{s^{2}+0.0422 s+0.000444} & \frac{0.00163}{s^{2}+0.0422 s+0.000444} \\
\frac{0.00163}{s^{2}+0.0422 s+0.000444} & \frac{0.015 s+0.002573}{s^{2}+0.0422 s+0.000444}\end{array}\right]$} \\
\hline
\end{tabular}

$\left.\mathrm{cm}^{3} / \mathrm{v} \cdot \mathrm{sec}\right) . \mathrm{V} 1, \mathrm{~V}_{2}$ are the voltage applied to the pumps $(0 \mathrm{~V}-5 \mathrm{~V})$.

The state space model and transfer function model is obtained around the operating points using Jacobian linearization.

State equation is,

$\dot{\mathrm{X}}=\mathrm{AX}+\mathrm{BU}$

where $\mathrm{X}$ is the states of the process $\left[\mathrm{h}_{1}, \mathrm{~h}_{2}\right]$ and $\mathrm{U}$ is the input vector of process $\left[\mathrm{V}_{1}, \mathrm{~V}_{2}\right]$. The $\mathrm{A}, \mathrm{B}$ matrixes are the state matrix and input matrix of the state space model.

$\left[\begin{array}{c}\frac{d h_{1}}{d t} \\ \frac{d h_{2}}{d t}\end{array}\right]=\left[\begin{array}{ll}\frac{\partial f_{1}}{\partial h_{1}} & \frac{\partial f_{1}}{\partial h_{2}} \\ \frac{\partial f_{2}}{\partial h_{1}} & \frac{\partial f_{2}}{\partial h_{2}}\end{array}\right]\left[\begin{array}{l}h_{1} \\ h_{2}\end{array}\right]+\left[\begin{array}{ll}\frac{\partial f_{1}}{\partial V_{1}} & \frac{\partial f_{1}}{\partial V_{2}} \\ \frac{\partial f_{2}}{\partial V_{1}} & \frac{\partial f_{2}}{\partial V_{2}}\end{array}\right]\left[\begin{array}{c}V_{1} \\ V_{2}\end{array}\right]$

where $f_{1}$ is the function $d_{1} / d t, f_{2}$ is the fucntion $\mathrm{dh}_{2} / \mathrm{dt}$. The output equation of state space model is given below,

$$
\mathrm{Y}=\mathrm{C} \mathrm{X}+\mathrm{D} \mathrm{U}
$$

where $\mathrm{Y}$ is the output vector $\left[\mathrm{h}_{1}, \mathrm{~h}_{2}\right], \mathrm{C}$ is the output matrix, D is the feedforward input matrix.

$$
\left[\begin{array}{l}
Y_{1} \\
Y_{2}
\end{array}\right]=\left[\begin{array}{ll}
1 & 0 \\
0 & 1
\end{array}\right]\left[\begin{array}{l}
h_{1} \\
h_{2}
\end{array}\right]+\left[\begin{array}{ll}
0 & 0 \\
0 & 0
\end{array}\right]\left[\begin{array}{c}
\mathrm{V}_{1} \\
\mathrm{~V}_{2}
\end{array}\right]
$$

Where $Y_{1}, Y_{2}$ are the outputs of the TICFT process. The state space model is converted into transfer function model which is tabulated for $3 \mathrm{X}$ 3operating condtions.

\section{DECENTRALIZED CONTROLLER}

The interaction between input and output is analyzed using Relative gain array. Then, the decentralized PI controller is designed for diagonal element using ziegler Nichols method and then the diagonal controllers are detuned.

$\operatorname{Gc}(\mathrm{s})=\left[\begin{array}{cc}k c_{11}\left(1+\frac{1}{\tau_{11} s}\right) & 0 \\ 0 & k c_{22}\left(1+\frac{1}{\tau_{22} s}\right)\end{array}\right]$

$\operatorname{diag}\left[k c_{i i}\right]=k c_{i i} / F ; \quad \operatorname{diag}\left[\tau_{i i}\right]=\tau_{i i} F$; where $\mathrm{i}=1,2$. The recommended value of detuning parameter ' $F$ ' is between 2 to 5 .

\section{CENTRALIZED PI, FRACTIONAL ORDER PI CONTROLLER}

\subsection{Centralized PI Controller}

The centralized PI Controller is designed for TICFTL process using the gain array of the plant. The gain matrix of operating region is used to find the centralized PI controller parameter (Davison EJ 1976).

$\mathrm{Kc}=\delta_{i}[G(s=0)]^{-1}$

$\mathrm{K}_{\mathrm{i}}=\varepsilon_{i}[G(s=0)]^{-1}$

The controller tuning parameters $\delta_{i}, \varepsilon_{\mathrm{i}}$ are called as rough tuning parameters. This parameter adjusted to change the controller gain of centralized PI controller.

\subsection{Centralized Fractional Order PI Controller}

The fractional order integrator is included in the conventional FOPI controller to improve the performance of PI controller. 
$\mathrm{Gc}(\mathrm{s})=\left[\begin{array}{cc}\left(k p_{11}+\frac{k_{i 11}}{s^{\lambda}}\right) & k c_{11}\left(1+\frac{1}{\tau_{11} s^{\lambda}}\right) \\ k c_{11}\left(1+\frac{1}{\tau_{11} s^{\lambda}}\right) & k c_{22}\left(1+\frac{1}{\tau_{22} s}\right)\end{array}\right]$

Consider the transfer function model and gain matrix of operating point 33 (Tank1/Regime 3, Tank2 /Regime 3),

$G_{p}(s)=\left[\begin{array}{ccc}\frac{0.0121 s+0.002686}{s^{2}+0.0422 s+0.000444} & \frac{0.00163}{s^{2}+0.0422 s+0.000444} \\ \frac{0.00163}{s^{2}+0.0422 s+0.000444} & \frac{0.015 s+0.002573}{s^{2}+0.0422 s+0.000444}\end{array}\right]$

The gain array at steady state is,

$\left[G_{\mathrm{p}}(0)\right]_{33}=\left[\begin{array}{ll}6.05 & 3.26 \\ 3.26 & 5.78\end{array}\right]$

The inverse of gain matrix,

$\left[G_{\mathrm{p}}(0)\right]^{-1}=\left[\begin{array}{cc}0.2378 & -0.134 \\ -0.134 & 0.2489\end{array}\right]$

The complex centralized problem is simplified and control scheme is given below,

$G_{c}(s)=\left[\begin{array}{cc}0.2378 * \delta_{\mathrm{f}}+\frac{0.2378 * \varepsilon_{\mathrm{f}}}{s^{\lambda}} & -0.134 * \delta_{\mathrm{f}}-\frac{0.134 *_{\mathrm{f}}}{s^{\lambda}} \\ -0.134 * \delta_{\mathrm{f}}-\frac{0.134 * \varepsilon_{\mathrm{f}}}{s^{\lambda}} & 0.2489 * \delta_{\mathrm{f}}+\frac{0.2489 * \varepsilon_{\mathrm{f}}}{s^{\lambda}}\end{array}\right]$

The parameters $\left(\delta_{\mathrm{f}}, \varepsilon_{\mathrm{f}}\right)$ are the tuning parameters of Centralized FOPI controller. The tuning parameter $\delta_{\mathrm{f}}$ change the proportional gain of centralized FOPI controller and parameter $\varepsilon_{\mathrm{f}}$ adjust the integral gain of centralized FOPI controller. The order of integrator is ' $\lambda$ '. The centralized FOPI controller parameters are obtained optimally to achieve minimum integral time absolute error (ITAE).

\subsection{Genetic Algorithm Based Controller Tuning}

Genetic algorithm is most widely used optimization algorithm to solve nonlinear multimodal problems. Many researchers have used genetic algorithm (GA) for tuning the controller with integral error criteria as an objective function. In this paper, the centralized fractional order FOPI controller is tuned using matlab genetic algorithm tool box (Houck et.al 1995)

Steps involved in Genetic optimization algorithm

Step 1: Initialize the objective function

$$
J=w_{1} \int_{0}^{\infty} \mathrm{t}\left|h_{1 s p}(t)-h_{1}(t)\right| \mathrm{d} \mathrm{t}+\mathrm{w}_{2} \int_{0}^{\infty} \mathrm{t}\left|h_{2 s p}(t)-h_{2}(t)\right| \mathrm{dt}
$$

Minimize J, subject to

$$
\delta_{\mathrm{f}}{ }^{\min } \leq \delta_{f} \leq \delta_{\mathrm{f}}{ }^{\max } ; \varepsilon_{f}{ }^{\min } \leq \varepsilon_{f} \leq \varepsilon_{f}{ }^{\max } ;
$$$$
\lambda^{\min } \leq \lambda \leq \lambda^{\max }
$$

Where ' $\mathrm{h}_{1 \mathrm{sp}}$ ', ' $\mathrm{h}_{2 \mathrm{sp}}$ ' are the reference set point of $1^{\text {st }}$ loop and $2^{\text {nd }}$ loop. The ' $h_{1}(t)$ ' ' $h_{2}(t)$ ' are the liquid level of the $1^{\text {st }}$ tank and $2^{\text {nd }}$ tank. The $\mathrm{w}_{1}, \mathrm{w}_{2}$ are the weigtage of sub objective function. $\left(\mathrm{W}_{1}=\mathrm{W}_{2}=0.5\right)$ Step 2: The population size is fixed as 20 , number of iteration is fixed as 100 and other optimization parameters such as mutation, cross over parameters are selected as 0.8 and constraint dependent.

Step 3: The fitness values objective functions is obtained based on rank based scaling function.

Step 4: At each iteration the control vectors are updated to achieve minimum value of objective function $\mathrm{J}$.

Step 5: The selection function is fixed as stochastic uniform function and the reproduction function such as elite count and cross over fraction is set as $0.05,0.8$. The initial guess for optimization problem for three cases such as decentralized PI, centralized PI and centralized FOPI is given below, case i: For decentralized PI controller, the controller parameters for each diagonal transfer function model is obtained using Z-N tuning formula and then, detuning factor $F_{1}, F_{2}$ fixed in between the range of 2-5.

Case ii; For the centralized controller, the initial guess of tuning parameters $\delta_{i}$ fixed between 0.1 to 50 and $\varepsilon$ fixed between 0.01 to 10 .

Case iii: For the centralized FOPI controller, the parameters are fixed as $\delta_{\mathrm{f}} \in[0.1,50]$, $\varepsilon_{\mathrm{f}} \in[0.01,10], \lambda \in[0.1,1.2]$.

Step 6: Run the optimization until the minimum objective function is obtained. The stopping criteria for the optimization are maximum iteration or minimum value convergence.

The controller parameters for objective functions $\mathrm{J}$ are obtained using GA and the obtained parameters are tabulated in Table. 4, 5. The block diagram of GA based decentralized/centralized controller tuning for TITO system is shown in Fig.3.

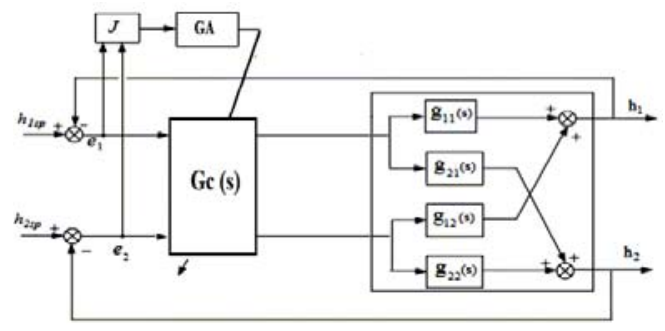

Fig. 3. Tuning of centralised PI/FOPI control parameter tuning using GA.

\subsection{Fuzzy Based Gain Scheduling Method}

The centralized PI controller designed for 3 X 3 operating points. The controller around one 
S. K. Lakshmanaprabu et al. / JAFM, Vol. 10, Special Issue, pp. 23-32, 2017.

Table 4 Optimal GA based controller parameter for 3-3 operating point region.

\begin{tabular}{|c|cc|}
\hline Operating point (3-3) & {$\left[\begin{array}{cc}\frac{0.0121 s+0.002686}{s^{2}+0.042 s+0.00044} & \frac{0.00163}{s^{2}+0.042 s+0.00044} \\
\frac{0.00163}{s^{2}+0.042 s+0.00044} & \frac{0.015 s+0.002573}{s^{2}+0.042 s+0.00044}\end{array}\right]$} \\
\hline Decentralized PI Controller & {$\left[\begin{array}{cc}0.721+\frac{0.0066}{s} & 0 \\
0 & 0.814+\frac{0.0075}{s}\end{array}\right]$} \\
\hline Centralized PI Controller & {$\left[\begin{array}{cc}0.2433+\frac{0.0046}{s} & -0.1541-\frac{0.0029}{s} \\
-0.1541-\frac{0.0029}{s} & 0.254+\frac{0.0048}{s}\end{array}\right]$} \\
\hline Centralized FOPI controller & {$\left[\begin{array}{rr}0.3439+\frac{0.004}{s^{1.051}} & -0.2179-\frac{0.0026}{s^{1.051}} \\
-0.2179-\frac{0.0026}{s^{1.051}} & 0.359+\frac{0.0042}{s^{1.051}}\end{array}\right]$} \\
\hline
\end{tabular}

operating region cannot produce satisfactory performance for complete process because of change in process gain and time constant. In order to accommodate nonlinearity, the gain scheduler is used in controller to vary the controller parameters according to different operating condition. The PI controller for each operating regions are combined using fuzzy gain scheduling method. The trapezoidal membership function is chosen and fuzzy rules are developed based on the operating regions. The tank levels are the input for fuzzy gain scheduler and controller parameters such that proportional gain $\left(\mathrm{Kp}_{11}, \mathrm{~K}_{\mathrm{p} 12}, \mathrm{~K}_{\mathrm{p} 21}, \mathrm{Kp}_{22}\right)$, integral gain $\left(\mathrm{Ki}_{11}, \mathrm{~K}_{\mathrm{i} 12}, \mathrm{~K}_{\mathrm{i} 21}, \mathrm{Ki}_{22}\right)$ are the outputs of the fuzzy gain scheduler. The memberships functions and rule viewer of fuzzy gain scheduler is shown in figs. 4,5 .

Table 5 Optimal GA based decentralized PI controller parameter for minimum ITAE

\begin{tabular}{|c|c|c|c|c|}
\hline & \multicolumn{4}{|c|}{ Decentralized PI } \\
\hline Regime & \multicolumn{3}{|l|}{} \\
\hline & Kp1 & Ki1 & Kp2 & Ki2 \\
\hline $1-1$ & 3.19 & 0.48 & 3.24 & 0.482 \\
\hline $1-2$ & 1.88 & 0.29 & 1.94 & 0.412 \\
\hline $1-3$ & 2.1 & 0.23 & 2.6 & 0.31 \\
\hline $2-1$ & 1.94 & 0.412 & 1.88 & 0.29 \\
\hline $2-2$ & 1.42 & 0.092 & 1.53 & 0.104 \\
\hline $2-3$ & 0.621 & 0.0136 & 0.54 & 0.0113 \\
\hline $3-1$ & 2.6 & 0.31 & 2.1 & 0.23 \\
\hline $3-2$ & 0.54 & 0.0113 & 0.621 & 0.0136 \\
\hline $3-3$ & 0.721 & 0.0066 & 0.814 & 0.0075 \\
\hline
\end{tabular}

Table 6 Optimal GA based controller parameter for minimum ITAE

\begin{tabular}{|c|c|c|c|c|c|}
\hline & \multicolumn{2}{|c|}{$\begin{array}{c}\text { Centralized } \\
\text { PI }\end{array}$} & \multicolumn{3}{c|}{ Centralized } \\
\hline Regime & $\delta_{i}$ & $\varepsilon_{\mathrm{i}}$ & $\delta_{\mathrm{f}}$ & $\varepsilon_{\mathrm{f}}$ & $\lambda$ \\
\hline $1-1$ & 20.76 & 0.85 & 20.51 & 0.84 & 0.991 \\
\hline $1-2$ & 15.76 & 0.44 & 16.51 & 0.56 & 0.979 \\
\hline $1-3$ & 9.76 & 0.34 & 10.51 & 0.26 & 0.982 \\
\hline $2-1$ & 15.76 & 0.44 & 16.51 & 0.56 & 0.979 \\
\hline $2-2$ & 2.76 & 0.248 & 2.91 & 0.024 & 1.04 \\
\hline $2-3$ & 1.76 & 0.14 & 1.97 & 0.02 & 1.03 \\
\hline $3-1$ & 9.76 & 0.34 & 10.51 & 0.26 & 0.982 \\
\hline $3-2$ & 1.76 & 0.14 & 1.97 & 0.02 & 1.03 \\
\hline $3-3$ & 0.906 & 0.017 & 1.281 & 0.015 & 1.051 \\
\hline
\end{tabular}

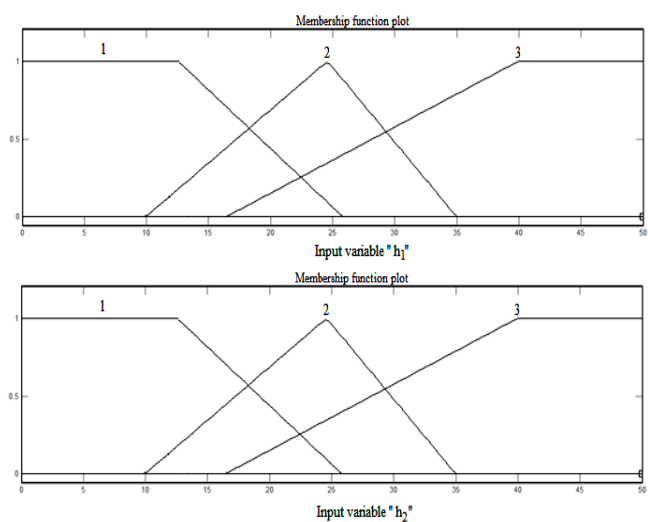

Fig. 4. Membership function of fuzzy gain scheduler. 


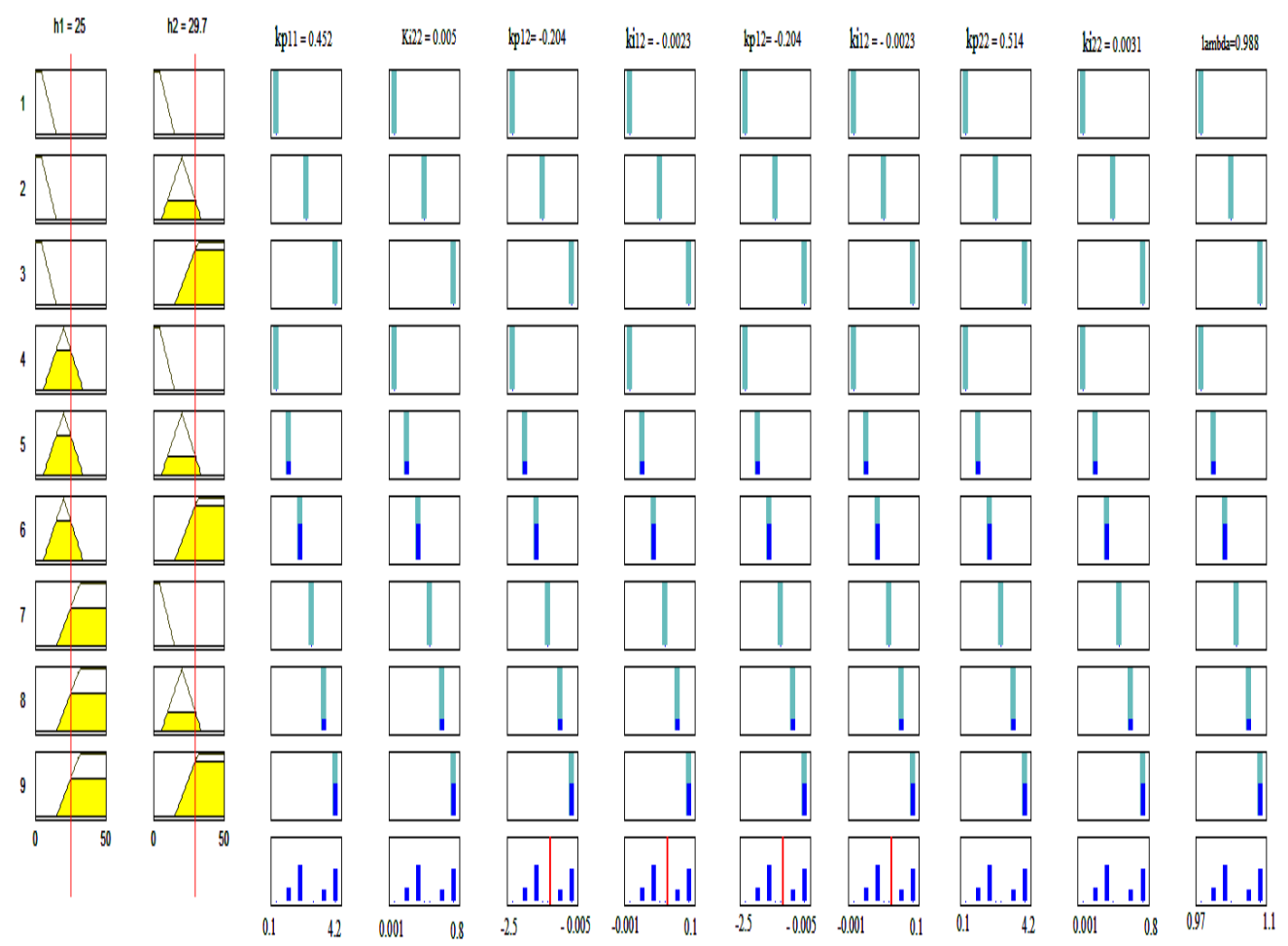

Fig. 5. Fuzzy Rule viewer of fuzzy gain scheduler.

The membership function and fuzzy rules are selected based on the operating condition. The adaptive centralized PI/FOPI controller is shown in Fig. 6.

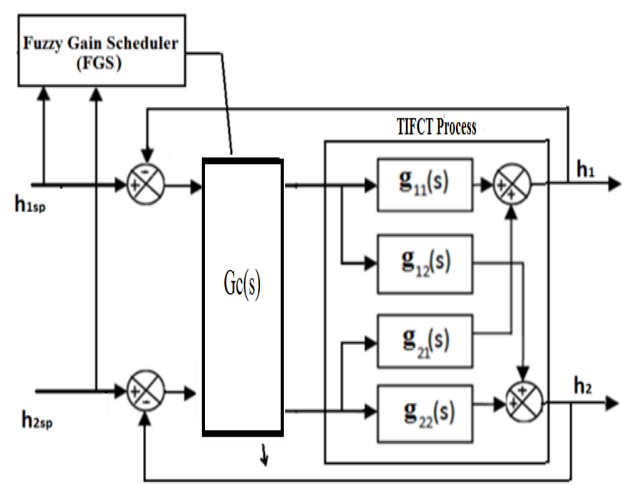

Fig. 6. Adaptive Centralized PI/ FO-PI controller.

\section{SIMULATION RESULTS ANALYSIS}

In the proposed work, the TICFTL process model is developed based on the mass balance equation. The open loop data was generated and the operating regimes are selected from the input output characteristics. The linear state space model is developed and converted into transfer function model. The decentralized PI, centralized PI, FOPI controller parameters are tuned using GA. The multivariable controller design is treated as multi objective optimization problem. The local linear controllers are combined using fuzzy gain scheduling method.

The servo and regulatory response of adaptive decentralized PI, centralized PI, centralized FOPI controllers responses are compared and shown in Figs. 7,8,9,10. The setpoint changes are introduced to tank 1,2 at $1000 \mathrm{sec}$. The first tank level set point is fixed as $20 \mathrm{~cm}$ from $0 \mathrm{sec}$ to $2000 \mathrm{sec}$ and then the set point is changed at $2000 \mathrm{sec}$ from $20 \mathrm{~cm}$ to $15 \mathrm{~cm}$. The tank 2 level set point changes applied at each $1000 \mathrm{sec}$. The step change in set point changes $0-15 \mathrm{~cm}, 15-25 \mathrm{~cm}, 25-20 \mathrm{~cm}$ are applied at $0,1000,2000 \mathrm{sec}$.

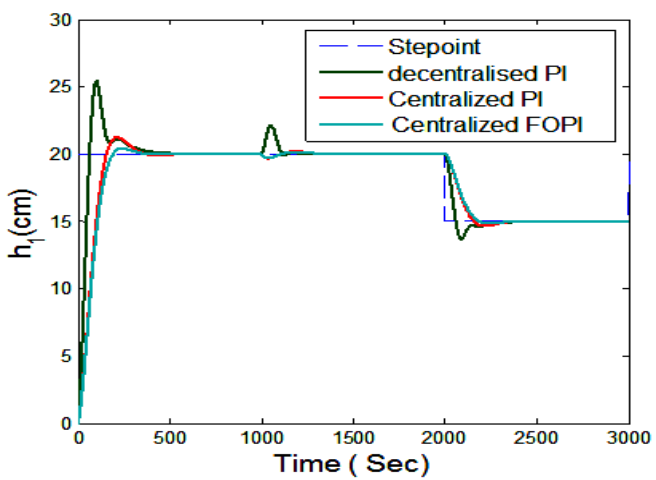

Fig. 7. Servo response of controller for TICFTL process (loop1). 
The servo tracking response of proposed centralized PI controller has better servo tracking and disturbance rejection response. The performance indexes are found and tabulated for comparison. It clearly shows that centralized PI controller has better servo tracking than decentralized PI, centralized FOPI controller.

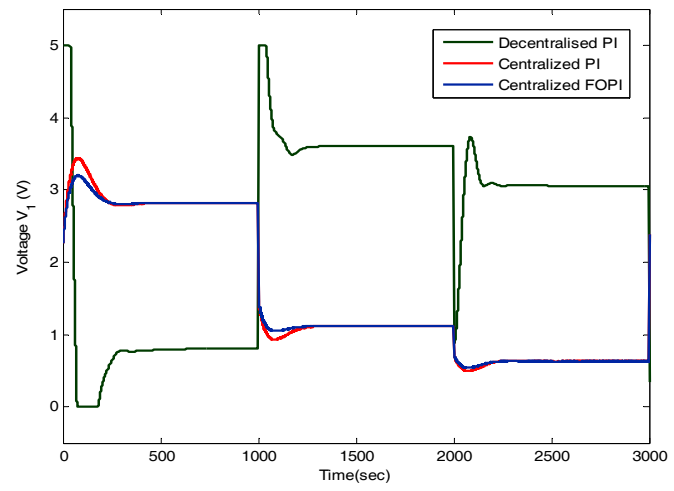

Fig. 8. Controller output voltage (manipulated input $\mathrm{V}_{1}$ ).

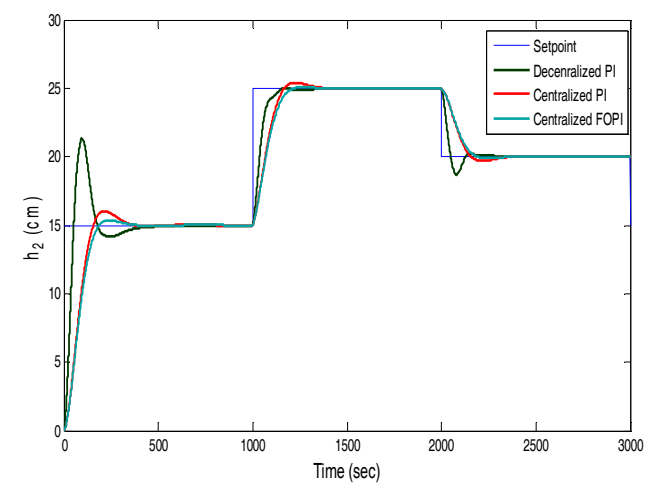

Fig. 9. Servo response of controller for TICFTL process (loop 2).

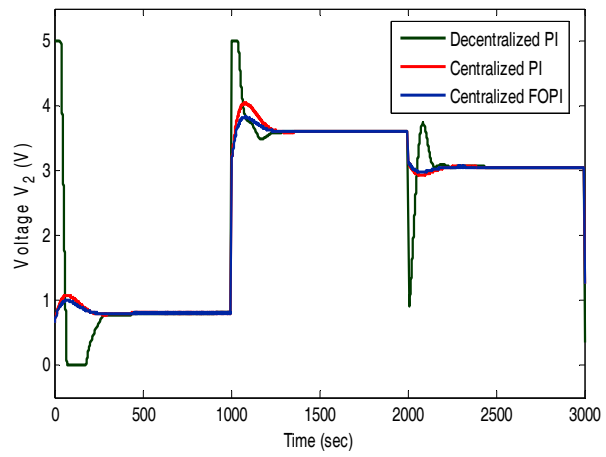

Fig. 10. Controller output voltage ( manipulated input $\mathrm{V}_{2}$ ).

The FOPI controller has three tuning parameters $\left(\delta_{\mathrm{f}}, \varepsilon_{\mathrm{f}}, \lambda\right)$, the order of FOPI controller $(\lambda)$ provides additional flexibility in controller tuning. It is believed that the fractional order integrator improves the performance of FOPI than PI controller. But the non integer integrator order results in the offset in the closed loop response which increases the settling time and offset. The FOPI controller provides better response when the order of integrator is equals to 1 . It can be easily inferred that the centralized PI controller has better performance than decentralized PI, centralized FOPI controller.

The proposed controller validated with servo tracking and regulatory responses. The centralized FOPI also produce reasonable controller response with faster settling time, minimum overshoot, but the integral error such as IAE, ITAE is larger for centralized FOPI controller. Because of the noninteger order integrator provides offset in the closed loop control performance.

Table 7 Comparison of decentralized PI, centralized PI, centralized FOPI controller

\begin{tabular}{|c|c|c|c|c|}
\hline & \multicolumn{2}{|c|}{ IAE } & \multicolumn{2}{c|}{ ITAE } \\
\hline & Loop1 & Loop2 & Loop1 & Loop2 \\
\hline $\begin{array}{c}\text { Decentralized } \\
\text { PI }\end{array}$ & 381.2 & 215.4 & 968.82 & 863.4 \\
\hline $\begin{array}{c}\text { Centralized } \\
\text { PI }\end{array}$ & 309.7 & 174.13 & 905.5 & 813.72 \\
\hline $\begin{array}{c}\text { Centralized } \\
\text { FOPI }\end{array}$ & 322.6 & 186.8 & 929.3 & 895.05 \\
\hline
\end{tabular}

\section{CONCLUSION}

The two interacting frustum conical tank interacting process is proposed and mathematical model was developed using mass balance equation. The interaction effect between input and output is analyzed for controller design. The genetic algorithm based centralized FOPI controller is presented and controller parameters are optimally tuned using genetic algorithm. The centralized FOPI controllers are compared with decentralized PI, centralized PI controller in terms of settling time, ISE, IAE, ITAE. From the simulation studies it is infer that the GA based centralized PI controller provides control response with faster settling time with minimum integral error criteria than centralized FOPI controller. It is concluded that, the fractional order PID may produce better response than the integer order PID controller, but FOPI controller does not improve the performance of closed loop control system than PI controller.

\section{REFERENCES}

Almeida Otacilio, M. and A. R. Antonio Coelho (2002). A Fuzzy logic method for auto tuning a PID Controller: SISO and MIMO Systems. 15th Triennial World Congress, Barcelona, Spain

Anandanatarajan, R., M. Chidambaram and T. Jayasingh (2006). Limitations of a PI controller for a first-order nonlinear process with dead time. ISA Transactions 45(2), 185-199.

Astrom, K. J., K. H. Johansson and Q. G. Wang 
(2002). Design of decoupled PI controllers for two-by-two system, IEEE Proceedings: Control Theory and Applications 149(1), 74-81.

Blanchett, T. P., G. C. Kember and R. Dubay (2000). PID gain scheduling using fuzzy logic. ISA Transactions 39, 317-325.

Concepcion, A. Monje, J. Antonio Calderon, M. Blas Vinagre, Y. Chen and V. Feliu (2004). On Fractional $P I^{\lambda}$ Controllers: Some Tuning Rules for Robustness to Plant Uncertainties. Nonlinear Dynamics 38, 369-381.

Davison, E. J. (1976). Multivariable tuning regulators: The feed forward and robust control of general servo mechanism problem. IEEE Transactions on Automatic Control 21(1), 3547.

Dhanalakshmi, R. and R. Vinodha (2013). Design of Control Schemes to Adapt PI controller for Conical Tank Process. International Journal of Advance Soft Computing. Appl. 5(3), 1-20.

Houck, C. R., J. Joines and M. Kay (1995). A genetic algorithm for function optimization: A MATLAB implementation. North Carolina State Univ Raleigh, NC, Tech. Rep. NCSU-IE-TR95-09.

Juan, G., F. Vazquez and F. Morilla (2012). Centralized multivariable control by simplified decoupling. Journal of process control 22, 1044-1062.

Kamala, N., N. Thyagarajan and S. Renganathan (2012). Multivariable control of nonlinear process using soft computing techniques. Journal of advances in information technology. $3(1), 1-9$

Morteza, M. (2014). A genetic-multivariable fractional order PID control to multi-inputmultioutput processes. Journal of Process Control 24 336-343.

Nithya, N. S., T. Balasubramanian and N. Anantharaman (2008). Design of Controller for Nonlinear Process Using Soft Computing, Instrumentation Science \& Technology 36(4), 437-450.

Pan, I. and S. Das (2013). Fractional Order Controller Tuning Using Swarm and Evolutionary Algorithms. Studies in
Computational Intelligence 438, 87-131.

Podlubny, I. (1999). Fractional-order systems and $\mathrm{P}^{\mathrm{I} \lambda} \mathrm{D}^{\delta}$ controllers. IEEE Transaction On Automatic Control 44(1), 208-213.

Priya, C. and P. Lakshmi (2011). Fractional Order Controller Design And Particle Swarm Optimization Applied to a Nonlinear System. Proc of IEEE International conference in information technology, Chennai 959-964.

Puneet, M., V. Kumar and K. P. S. Rana (2015). A fractional order fuzzyPID controller for binary distillation column control. Expert Systems With Applications.

Ravi, V. R and T. Thyagarajan (2014). Adaptive Decentralized PI Controller for Two Conical Tank Interacting Level System. Arabian Journal for Science and Engineering 39(12), 8433-8451.

Vijay Kumar, V., V. S. R. Rao and M. Chidambaram (2012). Centralized PI controller for interacting multivariable process by synthesis method. ISA Transactions 51, 400409.

Vijayalakshmi, S., D. Manmalli and G. Palanikumar (2014). Closed loop experimental validation of linear parameter varying model with adaptive PI controller for conical tank system. Control engineering and applied informatics 16(4), 1219.

Wei Der, C. (2007). A multi-crossover genetic approach to multivariable PID controllers tuning, Expert Systems with Applications. 33(3), 620-626.

Xiaoli, L., Q. Chen and F. Liu (2014).Centralized PI control for high dimensional multivariable systems based on equivalent transfer function. ISA Transaction 53, 1554-1561.

Yuling, S., Y. Sun and W. Xu (2014). Centralized PI/PID controller design for multivariable processes. Industrial \& Engineering Chemical Research 53, 10439-10447.

Zhao, Z. Y., M. Tomizuka and S. Isaka (1993). Fuzzy gain scheduling of PID controllers, IEEE Transaction Systems Man and Cybernetics 23 (5), 1392-1398 